\title{
Short-segment transverse myelitis lesions in a cohort of Latin American patients with neuromyelitis optica spectrum disorders
}

\author{
Edgar Carnero Contentti ${ }^{1}$. Vanessa Daccach Marques ${ }^{2}$ - Ibis Soto de Castillo ${ }^{3}$ - Verónica Tkachuk ${ }^{4}$. \\ Amilton Antunes Barreira ${ }^{2}$. Elizabeth Armas $^{5}$. Edson Chiganer ${ }^{6}$. Camila de Aquino Cruz ${ }^{2} \cdot$ José Luis Di Pace ${ }^{6}$. \\ Javier Pablo $\mathrm{Hryb}^{6} \cdot$ Carolina Lavigne Moreira $^{2} \cdot$ Carmen Lessa $^{6} \cdot$ Omaira Molina $^{3} \cdot$ Mónica Perassolo $^{6}$. \\ Arnoldo Soto $^{5} \cdot$ Alejandro Caride $^{1}$
}

Received: 23 January 2018 / Revised: 19 March 2018 / Accepted: 21 March 2018 / Published online: 22 May 2018

(c) International Spinal Cord Society 2018

\begin{abstract}
Study design Multicenter retrospective study.

Objectives The aim was to determine the frequency and magnetic resonance imaging (MRI) features of short-segment transverse myelitis (STM) in patients with neuromyelitis optica spectrum disorders (NMOSD) during a myelitis attack.

Setting Latin American diagnostic centres (Neuroimmunology Unit). A multicenter study from Argentina, Brazil and Venezuela was performed.

Methods Seventy-six patients with NMOSD were included. We analyzed 346 attacks and reviewed spinal cord MRIs performed within 30 days from spinal attack onset. Sagittal and axial characteristics on cervical and thoracic MRI (1.5 tesla) were observed. Demographics, clinical, serological, and disability data were collected.

Results Among the 76 patients with NMOSD, isolated STM was observed in $8 \%(n=6)$, multisegmental lesions (longitudinally extensive transverse myelitis (LETM) + STM) in 28\% ( $n=21 ; 13$ had at least one STM), LETM in $42 \%(n$ $=32)$, and normal spinal MRI in 22\% ( $n=17)$. However, isolated STM was increased by $10 \%$ in patients with NMOSD with spinal lesions ( 6 out of 59) with mean attacks of $2.5( \pm 0.83)$ and last follow-up expanded disability status scale (EDSS) of 3.1 ( \pm 2.63 ). Positive aquaporin 4 antibodies (AQP4-ab) were found in 50\%. Upper-cervical lesion was most frequently observed (5 out of 6). Myelitis was preceded by ON in all isolated patients with STM. Only one had a positive gadolinium lesion and none of these had asymptomatic spinal cord lesion.

Conclusion Isolated STM does not exclude NMOSD diagnosis. Therefore, APQ4-ab testing could be useful during a myelitis attack with STM.
\end{abstract}

Edgar Carnero Contentti

junior.carnero@hotmail.com

ecarnerocontentti@hospitalaleman.com

1 Neuroimmunology Unit, Department of Neuroscience Hospital Alemán, Buenos Aires, Argentina

2 Departamento de Neurociências e Ciências do Comportamento, Hospital das Clínicas da Faculdade de Medicina de Ribeirão Preto da Universidade de São Paulo, Sao Pablo, Brazil

3 Neurology Department, Hospital Universitario de Maracaibo, Maracaibo, Venezuela

4 Neurology Department, Hospital José de San Martin, Buenos Aires, Argentina

5 Neurology Department, Hospital Universitario de Caracas, Caracas, Venezuela

6 Neurology Department, Hospital Carlos G. Durand, Buenos Aires, Argentina

\section{Introduction}

Longitudinally extensive transverse myelitis (LETM) on magnetic resonance imaging (MRI) is defined as a lesion extending $\geq 3$ spinal segments. Recently, the 2015 International Panel for Neuromyelitis optica (NMO) spectrum disorders (NMOSD) Diagnosis criteria (IPND criteria) included LETM as a typical finding on spinal MRI [1]. Thus, an accurate and early identification of LETM (also lesions centrally-located on the axial plane) helps to differentiate NMOSD from multiple sclerosis (MS), especially in those patients who are positive for aquaporin 4 antibodies (AQP4-ab), given that this situation confirms the diagnosis of NMOSD by the 2015 IPND criteria $[1,2]$. Moreover, isolated or only one short-segment transverse myelitis (STM), defined as one lesion extending $<3$ spinal 


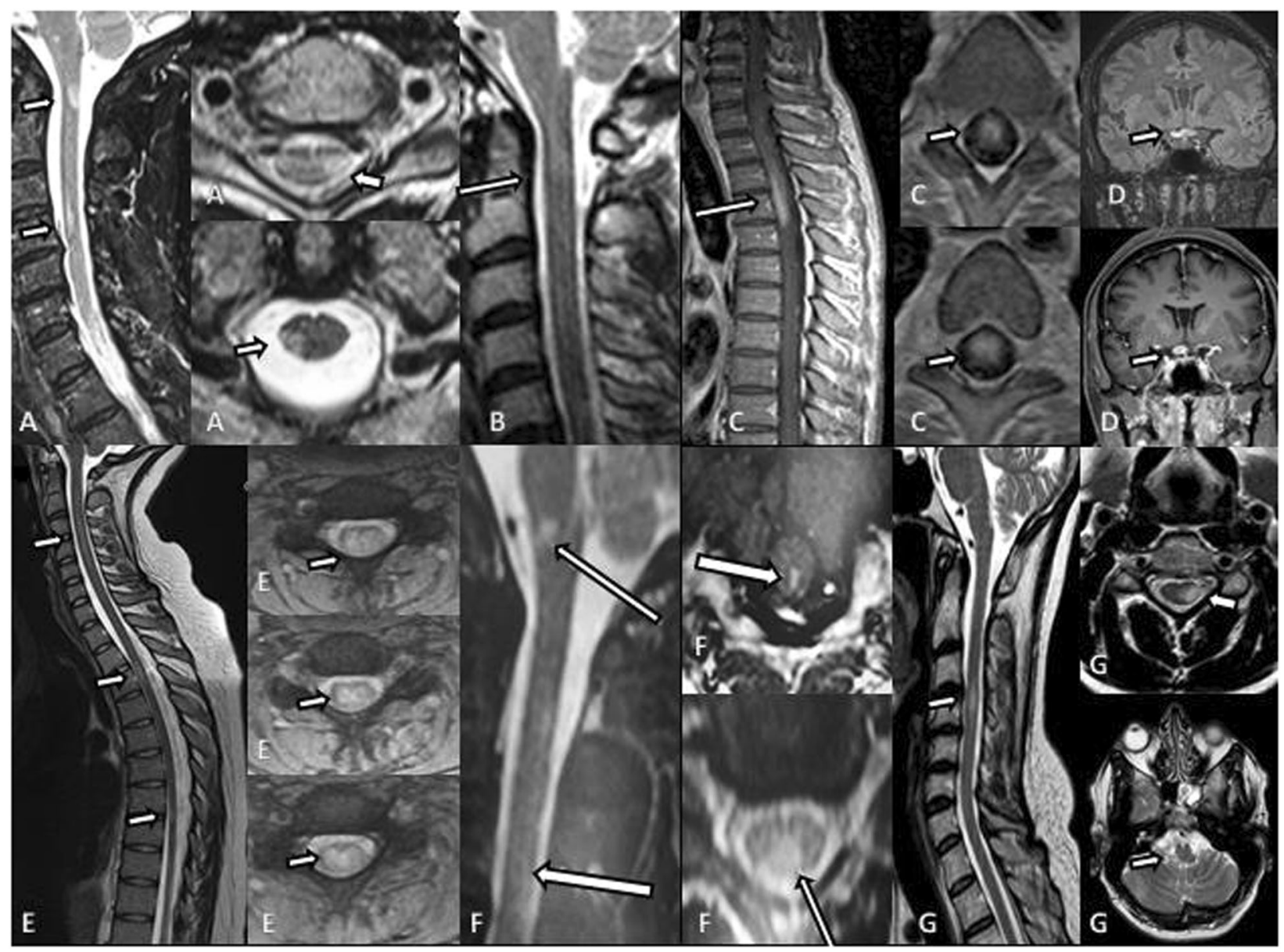

Fig. 1 MRI features of patients with NMOSD associated to STM. Two STM lesions (multisegmental STM lesions, A-arrow) on sagittal and axial (peripherally located with less than half of the cross-sectional cord area, A-arrow) T2-weighted MRI in a patient with NMOSD who was AQP4-ab-positive. STM lesion on C1 (B-arrow: 1-1.5 spinal segment long) in a patient with NMOSD who was AQP4-ab-negative (patient No 6). T1-hyperintensity post-contrast on sagittal (C-arrow: 1.5-2 spinal segment long) and axial (C-arrow: central, $\mathrm{H}$-type gray matter) images in a a patient with NMOSD who was AQP4-ab-negative with STM (patient No 4). Typical optic chiasm lesion (as initial manifestation, D-arrow) was found in a patient with NMOSD who was AQP4-ab-negative with STM on MRI (patient No
1). Multisegmental lesions (LETM on cervical and two discrete STM on thoracic MRI, E-arrow) on sagittal images and lateral / central (E -arrow) on axial images was observed in a patient with NMOSD who was AQP4-ab-positive. T2-hyperintensity extend into the medulla (axial image: bright spotty lesion, F-arrow) and STM (F-arrow) on cervical MRI (axial: posterior lesion) in a patient with NMOSD who was AQP4-ab-positive with STM (patient No 2). STM on C2-C3 is shown on T2-weighted sagittal (G-arrow: 1-1.5 spinal segment long) and axial (G-arrow: peripherally located) images. In addition, a patient with NMOSD who was AQP4-ab-positive with STM (patient No 5) also showed a dorsal medulla lesion (area postrema) on T2weighted axial segments, is highly suggestive of MS and these patients have low risk of developing NMOSD over time [1, 2]. However, there are few reports of patients with NMOSD presenting as STM during myelitis attacks, with similar results in different regions such as North America and Asia [3-5], but little is known about isolated STM in Latin American patients with NMOSD. Therefore, the aim of this study was to determine the frequency and MRI features of STM in a cohort of Latin American patients with NMOSD during a myelitis attack.

\section{Methods}

We retrospectively reviewed the medical records and MRI images of 76 patients with a diagnosis of NMOSD who were positive or negative for AQP4-ab from Argentina $(n=18)$, Brazil $(n=34)$, and Venezuela $(n=24)$. The diagnosis of NMOSD was performed according to the 2015 IPND criteria [1]. All patients fulfilled these criteria. We analyzed 281 out of 346 attacks (65 attacks were excluded due to normal spinal MRI) and reviewed spinal cord MRIs performed within 30 days from spinal attack onset. Sagittal and axial characteristics on cervical and thoracic MRI 


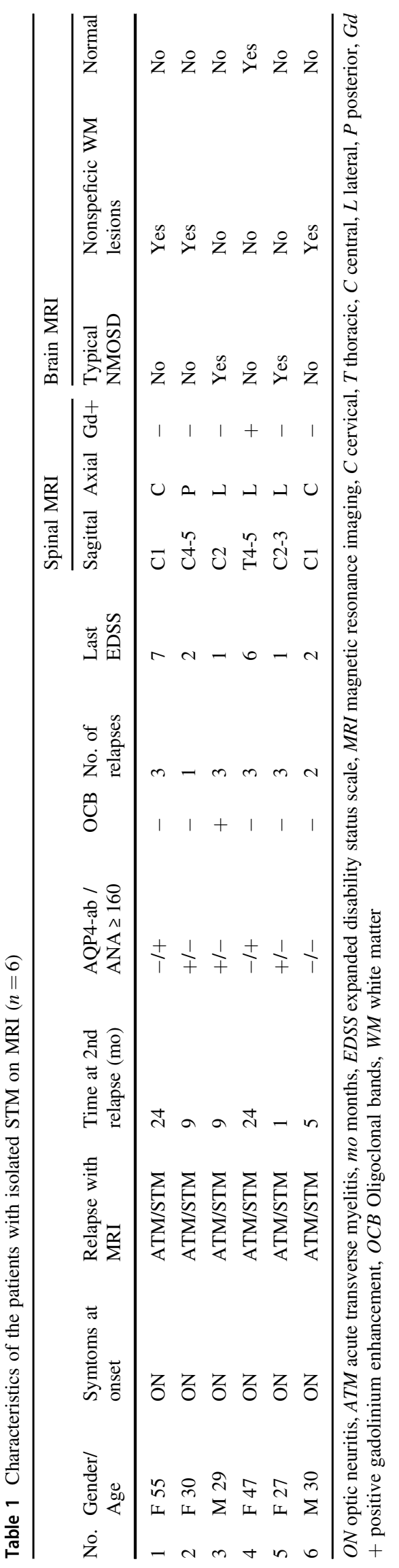

(1.5 tesla) were observed by at least one neurologist and one neuroradiologist (all of them with expertise in demyelinating diseases). In addition, brain and spinal MRI on T1-weighted with and without contrast enhancement, T2-weighted images, fluid-attenuated inversion recovery (FLAIR), and short tau inversion recovery sequences were evaluated in all patients. Lesions on spinal MRI included the extension (sagittal images), classified as isolated STM (only one lesion $<3$ spinal segments), LETM (lesions $\geq 3$ spinal segments) and multisegmental lesions (MSL, two or more lesions $<3$ and/or $\geq 3$ spinal segments in noncontiguous segment), and axial distribution (cross-sectional area) was classified as peripherally (laterally/posteriorly) located and centrally-located. Gadolinium enhancement lesions was classified as either present $(\mathrm{Gd}+)$ or not present (Gd-) on the T1 post-contrast images. Disability was measured by the expanded disability status scale (EDSS) at the last follow-up visit. This scale is probably the most commonly utilized assessment instrument that describes severity of disability in patients with MS, although it is also used in most demyelinating diseases of the spinal cord. The EDSS provides a total score on a scale that ranges from 0 (no disability) to 10 , based on a standard neurological examination of different functional systems (pyramidal, cerebellar, brainstem, sensory, bowel and bladder, visual, and cerebral (or mental) plus 'other'). AQP4-ab serostatus was assessed (particularly during the acute attack) by cell-based assay in $75 \%$ and by tissuebased indirect immunofluorescence assay in $25 \%$ as described previously [6]. Positive antinuclear antibodies (ANA, IIF using Hep-2 cell culture) were defined as $\geq 1 / 160$. Oligoclonal bands on cerebrospinal fluid were measured using isoelectric focusing. We excluded patients with normal spinal MRI $(n=17)$ and patients with LETM were included as a control group (for comparisons). All patients were treated with $1 \mathrm{~g}$ of intravenous methylprednisolone for 3-5 days in the acute phase.

This study was approved by the ethics committee of the Hospital Alemán de Buenos Aires, Argentina, and all participants signed an informed consent form before data collection.

\section{Statistical analysis}

Comparisons between STM patients and both MSL and LETM patients were performed using Chi square or Fisher's exact tests for categorical variables and $T$-test or Mann-Whitney $U$ test for continuous variables, when appropriate. Statistical analyses were performed using SPSS v 22 software. 


\section{Results}

In this study, 19 patients had at least one STM lesion. Among the 76 consecutive patients with NMOSD, isolated STM was observed in $8 \%(n=6)$, MSL $($ LETM + STM $)$ in $28 \%(n=21 ; 13$ had at least one STM), LETM in $42 \%$ $(n=32)$, and normal spinal MRI in $22 \%(n=17)$. However, STM was increased by $10 \%$ in patients with NMOSD with spinal lesions (6 out of 59). When considering the entire sample (76 patients with NMOSD), we observed that the mean lesion length on cervical MRI was $4.06( \pm 1.86)$ segments, STM was found in 10 patients, LETM in 36 patients, and normal in 30 patients. Centrally located lesions or H-type gray matter were observed in 40 patients and peripherally located in 6 attacks. Twenty-one patients had $\mathrm{Gd}+$ lesions. In thoracic MRI, the mean lesion length was $5.84( \pm 3.73)$ segments, STM was found in 8 patients, LETM in 33, and MSL in 4. On the axial plane, centrally located lesions were observed in 40 patients and peripherally located in 6 attacks. Sixteen patients had Gd+ lesions. Overall, in patients with STM $(n=19)$, the mean age at disease onset was $33.89( \pm 8.63)$, the mean attacks were $5.15( \pm 6.11)$, and EDSS at the last follow-up visit was $4.29( \pm 2.28)$. Both mean and median time between the first and the second attack was $12.05( \pm 16.80)$ months and 5 months, respectively. Positive AQP4-ab were found in $47.4 \%(n=9)$. Upper-cervical lesions (13 out of 19) were most commonly seen (two showed an extension to the medulla). None of these patients had asymptomatic spinal cord lesions. Seven patients had $\mathrm{Gd}+$ lesions on cervical MRI and five patients had $\mathrm{Gd}+$ lesions on thoracic MRI (Fig. 1).

As shown in Tables 1 and 2, optic neuritis (ON) preceded a myelitis event in all patients with isolated STM. Of the six patients with isolated STM, the mean attacks were $2.5( \pm 0.83)$ and EDSS at the last follow-up visit was 3.1 $( \pm 2.63)$. Positive AQP4-ab were observed in $50 \%(n=3)$. Upper-cervical lesion (5 out of 6) was most commonly seen in these patients (only one showed an extension to the medulla). None of these patients had asymptomatic spinal cord lesions. Comparison of demographic, laboratory, and radiological findings in patients with myelitis are summarized in Table 2.

\section{Discussion}

The present study showed that isolated STM may be observed in Latin American patients with NMOSD during a myelitis attack. Flanagan et al [3] (North America) found that $14 \%$ of patients with NMOSD had STM as an initial episode and $92 \%$ of these patients had subsequent LETM over time. Kim et al [4] (Asia) reported that STM was observed in $14.5 \%$ of their cohort as a first manifestation of NMOSD. We found that $10 \%$ of patients with NMOSD had isolated STM during a myelitis attack, but 32\% (19 out of 59) of our patients with spinal cord lesions had at least one STM lesion. Similar data were published by Jarius et al [7] (Europe) who observed that $7.3 \%$ of patients with NMOSD did not have LETM as first myelitis attack. STM as an initial manifestation of a first myelitis episode may delay the diagnosis of NMOSD and, therefore, delay the initiation of specific treatment to prevent a new attack. In addition, ON may have subtle symptom in its mild forms and could be underdiagnosed (sometimes the patients do not consult a physician). In this context, a myelitis event with STM during follow-up may be observed and testing AQP4-ab could be useful, especially in patients with a history of a typical ON for MS (NMOSD features) or optic chiasm lesion on MRI as evident in all of our isolated STM patients. The differential diagnoses are a challenge at disease onset, principally to distinguish NMOSD from MS. Likewise, an overlapping of symptoms among NMOSD and MS could occur at disease onset and misdiagnosis of MS may be seen $[3,8]$. The timing of MRI is very important because the length of the lesion depends on the timing of the MRI scan. Thus, both earlier (hours to days) and later (months after episode) imaging may have shown resolution or MSL [3]. We only included patients with spinal MRI performed within one month of a myelitis episode and we did not take into consideration only the first myelitis episode in each case. Therefore, this cohort could have contamination from old lesions and residual signal abnormality from an old myelitis attack that could explain some of the cases, particularly those with MSL. Nevertheless, in all our patients with isolated STM the myelitis attack was the first relapse on the spinal cord.

LETM on sagittal and centrally located lesions on axial images are a hallmark feature of NMOSD [1-10]. In this study, we observed that centrally-located lesions (on cervical MRI) were significantly more frequent in patients with LETM than in those with STM, but no statistical differences were found on thoracic MRI. In this line, both patients with LETM and those with MSL had significantly higher relapse rates than patients with STM. Interestingly, $67 \%$ of patients with STM had a relapse during the first year (as described previously in NMOSD [1]) and none of these had asymptomatic spinal cord lesions. In this cohort, we also observed a trend towards higher disability in patients with LETM compared to those with STM (5.4 vs. 3.1, $p=0.07$ ). Likewise, we recently reported that patients with LETM had a poorer functional prognosis compared to patients with STM [9]. Gd+ lesions previously were reported in up to $71 \%$ [10] of spinal cord lesions in patients with NMOSD and in this cohort we observed no cervical $\mathrm{Gd}+$ lesions of patients with STM compared to those with LETM (0 vs. 
Table 2 Comparison of demographic, laboratory, and radiological findings in patients with myelitis

\begin{tabular}{|c|c|c|c|c|c|}
\hline Characteristics & STM & LETM & MSL & $p$ value $^{\mathrm{a}}$ & $p$ value $^{\mathbf{b}}$ \\
\hline N No & 6 & 32 & 21 & & \\
\hline Mean age at onset $\mathrm{y},( \pm \mathrm{SD})$ & $36.3( \pm 11.3)$ & $41.2( \pm 13.3)$ & $34.1( \pm 10.1)$ & NS & NS \\
\hline Female no $(\%)$ & $4(67)$ & $28(87)$ & $19(90)$ & NS & NS \\
\hline \multicolumn{6}{|l|}{ Ethnicity (\%) } \\
\hline Caucasian & $4(67)$ & $18(56)$ & $15(72)$ & NS & NS \\
\hline Non-Caucasian & $2(33)$ & $14(44)$ & $6(28)$ & NS & NS \\
\hline Relapses & 15 & 144 & 122 & & \\
\hline Mean $( \pm \mathrm{SD})$ & $2.5( \pm 0.8)$ & $4.5( \pm 3.3)$ & $6.1( \pm 5.9)$ & 0.006 & 0.01 \\
\hline Median & 3 & 4 & 4 & 0.02 & 0.01 \\
\hline Time at second relapse ( $\mathrm{m}$, median) & 9 & 7 & 6 & NS & NS \\
\hline Last EDSS mean $( \pm \mathrm{SD})$ & $3.1( \pm 2.6)$ & $5.4( \pm 2.8)$ & $4.8( \pm 2.3)$ & 0.07 & NS \\
\hline \multicolumn{6}{|l|}{ Cervical MRI } \\
\hline Total segment lesions & 7 & 108 & 72 & & \\
\hline Mean length $( \pm$ SD) & $1.4( \pm 0.5)$ & $5.1( \pm 1.4)$ & $3.6( \pm 1.6)$ & 0.0001 & 0.0001 \\
\hline Median length & 1 & 5 & 3 & 0.0005 & 0.004 \\
\hline $\mathrm{Gd}+$ lesions & 0 of 5 & 15 of 21 & 6 of 20 & 0.007 & NS \\
\hline Axial topography LP/C & $3 / 2$ & $0 / 21$ & $3 / 17$ & 0.03 & NS \\
\hline \multicolumn{6}{|l|}{ Thoracic MRI } \\
\hline Total segment lesions & 2 & 176 & 91 & & \\
\hline Mean length $( \pm \mathrm{SD})$ & 2 & $7.3( \pm 3.9)$ & $4.3( \pm 2.7)$ & 0.0001 & 0.0001 \\
\hline Median length & 2 & 7.5 & 4 & 0.0001 & 0.0005 \\
\hline Gd + lesions & 1 of 1 & 11 of 24 & 7 of 20 & NS & NS \\
\hline Axial topography LP/C (No) & $1 / 0$ & $1 / 23$ & $4 / 12$ & NS & NS \\
\hline \multicolumn{6}{|l|}{ Paraclinical features no $(\%)$} \\
\hline AQP4-ab positive & $3(50)$ & $20(62)$ & $12(57)$ & NS & NS \\
\hline ANA positive & $2(33)$ & $5(16)$ & $6(28)$ & NS & NS \\
\hline
\end{tabular}

apatients with STM compared to those with LETM

b patients with STM compared to those with MSL

$M R I$ magnetic resonance imaging, $m$ months, $L P$ lateral/posterior lesion on axial images, $C$ central lesion on axial images, $A Q P 4-a b$ aquaporin 4 antibodies, $A N A$ antinuclear antibodies, $N S$ not significant
$71 \%, p=0.007)$. On the other hand, there were no statistical differences on gender, age, ethnicity, and paraclinical features between patients with STM and those with LETM, and between patients with STM and those with MSL.

We acknowledge several limitations in our study. This work was retrospective with a small sample and a possible selection bias. Moreover, AQP4-ab was not assessed in all patients with NMOSD with cell-based assay test and more patients could be AQP4-ab-positive as recommended by the 2015 IPND criteria. In addition, myelin oligodendrocyte glycoprotein antibodies (MOG-ab) were not tested in this sample, but should be performed especially in patients who are AQP4-ab-negative, as recommended in several studies $[1,3,4,6-8]$. However, as there were no data on any large multicenter cohort of Latin American patients we assessed our cohort and add our data to the international data set in order to compare with previously published results from Asia, Europe, and North America.
In conclusion, our findings suggest that isolated STM is a rare manifestation in patients with NMOSD but does not exclude the diagnosis of NMOSD. Therefore, APQ4-ab testing could be useful during a myelitis attack with STM on spinal cord MRI.

Funding This research received no specific grant from any funding agency in the public, commercial, or non-profit sectors.

Author contributions ECC was responsible for conception and design, acquisition of data, analysis and interpretation of data, drafting the manuscript, revising it for intellectual content, and finally for the final approval of the completed manuscript. VDM was responsible for acquisition of data, drafting the manuscript, revising it for intellectual content, and finally for the final approval of the completed manuscript. ABA, CdAC , CLM, ISdC, EA, VT, EC, JLDP, JPH, CL, OM, MP, $\mathrm{AS}$, and $\mathrm{AC}$ were responsible for acquisition of data, revising it for intellectual content, and finally for the final approval of the completed manuscript.

Conflict of interest The authors declare that they have no conflict of interest. 


\section{References}

1. Wingerchuk DM, Banwell B, Bennett JL, Cabre P, Carroll W, Chitnis $\mathrm{T}$, et al. International consensus diagnostic criteria for neuromyelitis optica spectrum disorders. Neurology. 2015;85:177-89.

2. Scott TF, Frohman EM, De Seze J, Gronseth GS, Weinshenker BG. Evidence based guideline: clinical evaluation and treatment of transverse myelitis: Report of the Therapeutics and Technology Assessment Subcommittee of the American Academy of Neurology. Neurology. 2011;77:2128-34.

3. Flanagan EP, Weinshenker BG, Krecke KN, Lennon VA, Lucchinetti CF, McKeon A, et al. Short myelitis lesions in aquaporin-4-IgG-positive neuromyelitis optica spectrum disorders. JAMA Neurol. 2015;72:81-7.

4. Huh SY, Kim SH, Hyun JW, Jeong IH, Park MS, Lee SH, et al. Short segment myelitis as a first manifestation of neuromyelitis optica spectrum disorders. Mult Scler. 2017;23:413-9.
5. Ortega MR, Tornes L, Rammohan KW. NMO spectrum presenting as partial myelitis. Mult Scler. 2013;19:252-3.

6. Waters P, Reindl M, Saiz A, Schanda K, Tuller F, Kral V, et al. Multicentre comparison of a diagnostic assay: aquaporin- 4 antibodies in neuromyelitis optica. J Neurol Neurosurg Psychiatry. 2016;87:1005-15.

7. Jarius S, Ruprecht K, Wildemann B, Kuempfel T, Ringelstein M, Geis $\mathrm{C}$, et al. Contrasting disease patterns in seropositive and seronegative neuromyelitis optica: a multicenter study of 175 patients. J Neuroinflamm. 2012;9:14.

8. Juryńczyk M, Craner M, Palace J. Overlapping CNS inflammatory diseases: differentiating features of NMO and MS. J Neurol Neurosurg Psychiatry. 2015;86:20-5.

9. Carnero Contentti E, Hryb JP, Diego A, Di Pace JL, Perassolo M. Etiologic spectrum and functional outcome of the acute inflammatory myelitis. Acta Neurol Belg. 2017;117:507-13.

10. Tackley G, Kuker W, Palace J. Magnetic resonance imaging in neuromyelitis optica. Mult Scler. 2014;20:1153-64. 